\title{
Use of Patient Reported Outcomes to Inform Supportive Care for Geriatric Oncology Patients.
}

Cuthbert, C. ${ }^{1}$, Hemmelgarn, B. ${ }^{2}$, Boyne, D. ${ }^{2}$, Cheung, W. ${ }^{1,3}$

\section{Background}

Assessment of cancer patient symptoms and supportive care needs using Patient Reported Outcomes (PROs) is essential to patient centered care ${ }^{1}$.

- The collection of PROs is now a standard in many cancer organizations ${ }^{2}$.

- Data from PROs may help to inform specific supportive care strategies, however very little research exists on PROs in Geriatric Oncology.

\section{Aims}

- To determine differences in older versus younger cancer patients with respect to symptom severity and supportive care needs.

\section{Methods}

- Population cohort using administrative databases in Alberta, Canada.

- Adults with any cancer diagnosed between Jan 1, 2016 to Oct 23, 2017.

- Completed a PRO within +/- 60 days of diagnosis. $n=1310$

- PROs include:

1. Edmonton Symptom Assessment System (ESAS) ${ }^{3}$

2. Canadian Problem Checklist $(\mathrm{CPC})^{4}$

- $\quad$ Cohort was divided into Older (age $\geq$ 70) or Younger (age <70).

- Linear regression was used to determine predictors of high symptom burden.

\begin{tabular}{|c|c|c|c|}
\hline \multicolumn{4}{|c|}{ Results } \\
\hline \multicolumn{4}{|l|}{ Characteristics of Cohort $n=1310$} \\
\hline & Age $<70(n=911)$ & Age $>70(n=399)$ & Stat sig. \\
\hline \multicolumn{4}{|l|}{ Sex $\%$} \\
\hline Male & 46.5 & 53.8 & \\
\hline Female & 53.5 & 46.1 & \\
\hline Cancer site \% & & & $>.001$ \\
\hline CRC & 12.6 & 7.8 & \\
\hline Prostate & 7.6 & 8.0 & \\
\hline Breast & 23.1 & 9.3 & \\
\hline Lung & 21.7 & 31.6 & \\
\hline Hematological & 11.1 & 13.0 & \\
\hline All other cancers & 23.9 & 30.3 & \\
\hline \multicolumn{4}{|l|}{ Cancer Stage \% } \\
\hline Local/Regional & 48.2 & 35.8 & $>.001$ \\
\hline Metastatic & 42.6 & 49.4 & \\
\hline Not applicable (hematology/missing) & 9.2 & 14.8 & \\
\hline Charlson Comorbidity Index Score & & & $>.001$ \\
\hline 0 & 52.1 & 27.8 & \\
\hline 1 & 27.6 & 24.6 & \\
\hline $2+$ & 20.3 & 47.9 & \\
\hline
\end{tabular}

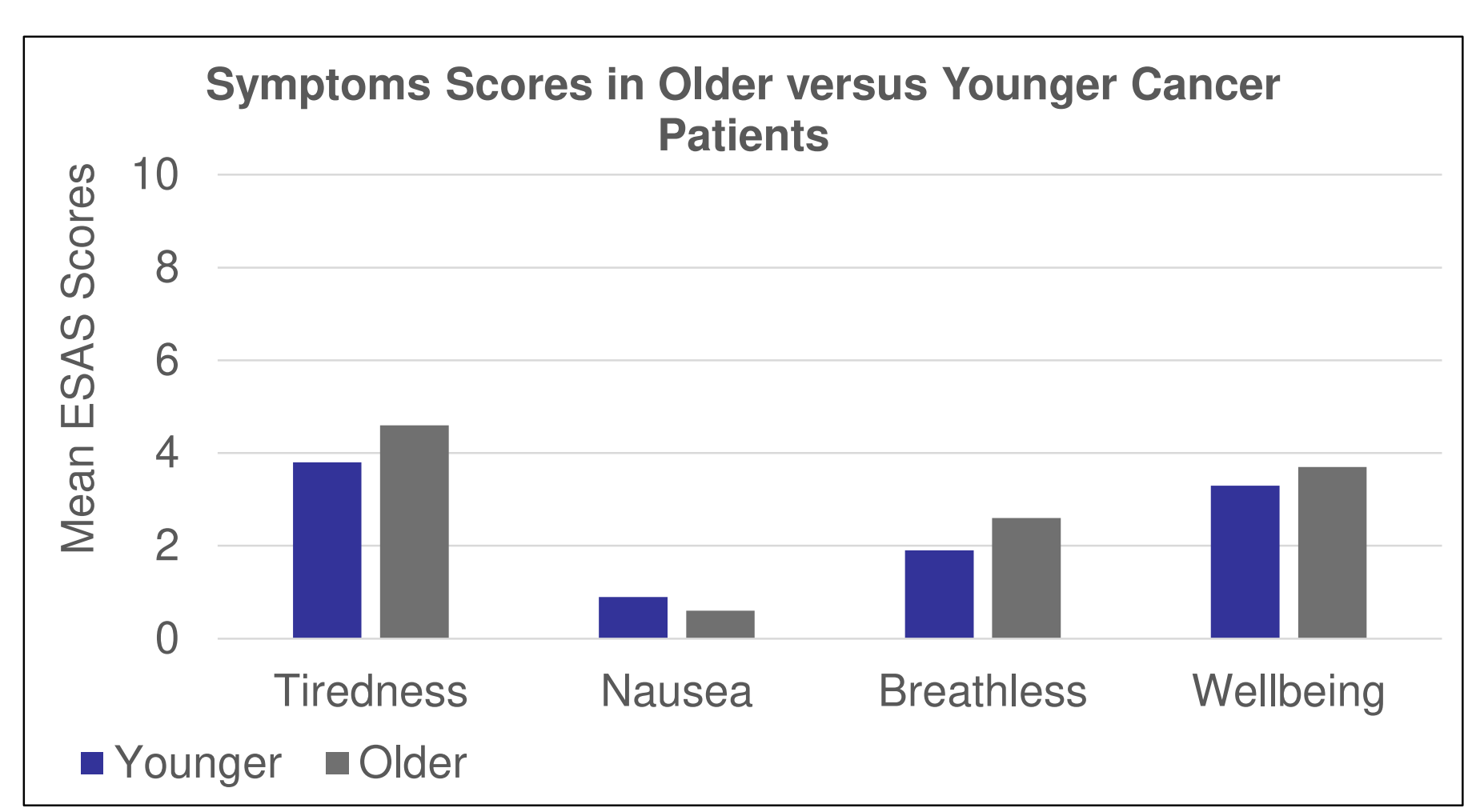

Figure 1: Differences in ESAS symptom scores $p<0.05$

Predictors of high ESAS Physical sub-score in Older patients included:

- Advanced stage $(\beta=.03)$. Breast $(\beta=-0.2)$, Colorectal $(\beta=-0.2)$ and Prostate $(\beta=-0.3)$ cancer were protective.

Predictors of high ESAS Emotional sub-score in Older patients included:

- Colorectal $(\beta=0.2)$ or Lung $(\beta=0.2)$ cancers. (all $p<0.05)$

Table 2: Top 5 Supportive Care Needs by Age Group

\begin{tabular}{|l|l|l|}
\hline & Age $<70$ \\
\hline Rank & Problem Needing Help With (\%reporting) \\
\hline 1 & Fears and worries (36) & Understanding illness/treatment (25) \\
\hline 2 & Understanding illness/treatment (27) & Fears and worries (25) \\
\hline 3 & Worry about family and friends (23) & Walking/mobility (23) \\
\hline 4 & Difficulties with sleep (22) & Difficulty with weight loss (21) \\
\hline 5 & Making treatment decisions (21) & Loss of strength (19) \\
\hline
\end{tabular}

\section{Conclusions}

- Symptoms and supportive care needs were different depending on age underscoring the need for age-specific supportive care interventions.

- PROs specific to older cancer patients should be investigated in the future to enhance supportive care. 